\title{
Treatment of unresectable malignant pleural mesothelioma in 2021: emerging standards in immunotherapy
}

\author{
Bailey G. Fitzgerald ${ }^{1}$, Lee M. Krug ${ }^{1,2}$ \\ ${ }^{1}$ Tisch Cancer Institute, Icahn School of Medicine at Mount Sinai, New York, NY 10029, USA. \\ ${ }^{2}$ AstraZeneca, Gaithersburg, MD 20878, USA.
}

Correspondence to: Dr. Lee M. Krug, Icahn School of Medicine at Mount Sinai, 1 Gustave L. Levy Pl., New York, NY 10029, USA. E-mail: lee.krug@mssm.edu

How to cite this article: Fitzgerald BG, Krug LM. Treatment of unresectable malignant pleural mesothelioma in 2021: emerging standards in immunotherapy. J Cancer Metastasis Treat 2021;7:54. https://dx.doi.org/10.20517/2394-4722.2021.97

Received: 15 Apr 2021 First Decision: 25 May 2021 Revised: 9 Jun 2021 Accepted: 29 Jun 2021 First online: 5 Aug 2021

Academic Editor: Lucio Miele Copy Editor: Xi-Jun Chen Production Editor: Xi-Jun Chen

\begin{abstract}
Patients with unresectable malignant pleural mesothelioma (MPM) have historically poor outcomes and treatment, and their treatments have been limited to palliative chemotherapy. Recent efforts to improve prognosis for these patients by adding angiogenesis inhibitors to chemotherapy led to significant benefits. However, the emergence of immunotherapy combinations for the front-line treatment has upended the standard of care and has led to the first new FDA approvals for the treatment of MPM in nearly two decades. This review aims to cover the main clinical trials in unresectable MPM with VEGF inhibitors and immunotherapy which have led to paradigm shifts in current practice. Ongoing clinical trials exploring the combination of chemo and immunotherapies show a great deal of promise, and continued support for ambitious, large-scale well-designed trials remain vital for defining the future outcomes of patients diagnosed with MPM.
\end{abstract}

Keywords: Malignant pleural mesothelioma, VEGF, chemotherapy, immunotherapy, PD-L1, CTLA-4

\section{INTRODUCTION}

Treatment of unresectable malignant pleural mesothelioma (MPM) remains a challenging problem, and outcomes are most often disappointing. In cases where a patient is considered as a surgical candidate, 
resection by pleurectomy or extra-pleural pneumonectomy can be offered, but recurrence and progression typically occur. Unfortunately, most patients present with advanced disease, and treatment is predominantly palliative. Traditional chemotherapy, up until recently the international standard of care for unresectable disease, provides only limited benefit. MPM is generally categorized as epithelioid or nonepithelioid, with sarcomatoid and mixed histologies falling under the non-epithelioid categorization. Epithelioid histology can respond somewhat better to treatment with traditional chemotherapy and has slightly better prognosis, while non-epithelioid histology is chemoresistant and progresses rapidly. Beyond histology, the field still lacks predictive biomarkers to identify patients who will derive the most benefit from a particular therapy or combination. Fortunately, recent studies with anti-VEGF monoclonal antibodies have demonstrated benefit when added to chemotherapy, and, even more significantly, the advent of immunotherapy has sparked a paradigm shift in the treatment of MPM. This review will contextualize the substantial impact of these therapies which will improve the treatment outcomes and quality of life for patients with MPM who previously had limited treatment options. Other targeted approaches will also be discussed in this issue of the journal. Continued support of robust clinical research will be indispensable in integrating these advances into the evolving standard of care and addressing the ongoing unmet need for these patients.

\section{CHEMOTHERAPY AND ANTI-ANGIOGENESIS}

Until recently, platinum-based chemotherapy with pemetrexed was the established standard for unresectable MPM; cisplatin plus pemetrexed remained the only FDA approved first-line treatment for MPM until 2020. This practice was founded largely on the results of the phase III EMPHACIS trial reported in 2003, in which 456 patients were randomized to receive pemetrexed in combination with cisplatin (CP) $v$ s. cisplatin alone ${ }^{[1]}$. The combination provided a nearly 3 -month survival benefit with a median OS at 12.1 months for CP vs. 9.3 months with cisplatin monotherapy. Response rate in the intention to treat population was also superior for the combination, at $41.3 \% v$ s. $16.7 \%$ for the cisplatin alone and time to progression improved to 5.7 months from 3.9 months. Although outcomes were better with combination chemotherapy, the fact that less than half of the patients treated with cisplatin plus pemetrexed had objective responses to treatment, and only $50 \%$ of the intervention arm survived to 1 year demonstrated the continued need for improved or adjunctive therapy options.

\section{VEGF receptor inhibitors}

Strategies utilizing the addition of a third agent to platinum-based chemotherapy have had mixed success. Twenty years ago, a series of pre-clinical studies provided compelling evidence suggesting a pivotal role for the VEGF/PDGF pathway in the pathogenesis of MPM, primarily by demonstrating that VEGF/VEGFR levels correlate with angiogenesis in MPM cell lines ${ }^{[2,3]}$. Armed with this rationale, trials incorporating the anti-VEGF monoclonal antibody bevacizumab were designed [Table 1]. Following promising anti-tumor efficacy in phase II trials, the combination of bevacizumab plus CP was evaluated in the Mesothelioma Avastin Cisplatin Pemetrexed Study (MAPS), a large, phase III randomized controlled trial conducted by the French thoracic oncology cooperative group, $\operatorname{IFCT}^{[4,5]}$. This study recruited patients with unresectable MPM who had not received prior chemotherapy. The primary endpoint of median overall survival (mOS) was significantly longer with the addition of bevacizumab to chemotherapy at 18.8 months $v s .16 .1$ months with chemotherapy alone, with a hazard ratio (HR) 0.77 favoring bevacizumab, despite a longer mOS in the $\mathrm{CP}$ arm than was seen in the EMPHACIS trial. Although a trend towards decreased benefit from bevacizumab addition was observed in patients whose tumors had epithelioid histology, no population identified by the authors within the subgroup analysis reliably predicted which patients benefit from the addition of bevacizumab to chemotherapy. The benefit in mOS in the bevacizumab arm of MAPS came at a cost of expected adverse events related to bevacizumab, including hypertension $(56.3 \%, 23 \% \geq$ grade 3$)$, cardiovascular events (61.7\%), thrombotic events (7.2\%), proteinuria (16.7\%) and hemorrhage (41.0\%). 
Table 1. Selected trials in utilizing anti-VEGF agents and immunotherapies the first-line treatment of unresectable or recurrent MPM

\begin{tabular}{|c|c|c|c|c|c|c|c|c|c|}
\hline Registry number & $\begin{array}{l}\text { Trial name/ } \\
\text { Group }\end{array}$ & Drug & Phase & Design & $\begin{array}{l}\text { Number of } \\
\text { patients }\end{array}$ & $\begin{array}{l}\text { Primary } \\
\text { endpoint (Met? } \\
\text { Y/N) }\end{array}$ & $\begin{array}{l}\text { mOS } \\
\text { (months) }\end{array}$ & $\begin{array}{l}\text { PFS } \\
\text { (months) }\end{array}$ & $\begin{array}{l}\text { ORR } \\
\text { (RECIST) }\end{array}$ \\
\hline \multicolumn{10}{|l|}{ Chemo + VEGF } \\
\hline NCT01064648 & SWOG S09505 & Cedirinib & ॥ & $\begin{array}{l}\text { Randomized; chemotherapy + cedirinib vs. } \\
\text { chemotherapy + placebo }\end{array}$ & 92 & $\operatorname{PFS}(Y)$ & $\begin{array}{l}10 \text { vs. } 8.5 \\
(H R=0.71)\end{array}$ & $\begin{array}{l}7.2 \text { vs. } 5.6 \\
(H R=0.71)\end{array}$ & $26 \%$ vs. $15 \%$ \\
\hline NCT01907100 & Lume Meso & Nintedinib & III & $\begin{array}{l}\text { Randomized; chemotherapy + nintedinib vs. } \\
\text { chemotherapy + placebo }\end{array}$ & 541 & $\operatorname{PFS}(N)$ & $\begin{array}{l}14.4 \text { vs. } 16.1 \\
(H R=1.12)\end{array}$ & $\begin{array}{l}6.8 \text { vs. } 7.0 \\
(H R=1.01)\end{array}$ & $45 \%$ vs. $43 \%$ \\
\hline NCT00651456 & $\begin{array}{l}\text { MAPS/IFCT- } \\
\text { GFPC-0701 }\end{array}$ & Bevacizumab & III & $\begin{array}{l}\text { Randomized; chemotherapy }+ \\
\text { bevacizumab vs. chemotherapy }+ \text { placebo }\end{array}$ & 448 & $\mathrm{OS}(\mathrm{Y})$ & $\begin{array}{l}18.8 \text { vs. } 16.1 \\
(H R=0.77)\end{array}$ & $\begin{array}{l}9.2 \text { vs. } 7.3 \\
(H R=0.61)\end{array}$ & NR \\
\hline \multicolumn{10}{|l|}{ Immunotherapy } \\
\hline NCT02899299 & CheckMate $743^{*}$ & Nivolumab + Ipilimumab & III & $\begin{array}{l}\text { Randomized; chemotherapy }+ \\
\text { bevacizumab vs. chemotherapy }\end{array}$ & 713 & $\operatorname{OS}(Y)$ & $\begin{array}{l}18.1 \text { vs. } 14.1 \\
(H R=0.74)\end{array}$ & 6.8 vs. 7.2 & $\begin{array}{l}40 \% \text { vs. } \\
43 \%\end{array}$ \\
\hline \multicolumn{10}{|l|}{ Chemo-immunotherapy } \\
\hline ACTRN12616001170415 & DREAM & $\begin{array}{l}\text { Cisplatin }+ \text { Pemetrxed }+ \\
\text { Durvalumab }\end{array}$ & ॥ & $\begin{array}{l}\text { Single arm; chemotherapy + durvalumab } \\
\text { followed by durvalumab maintenance }\end{array}$ & 55 & $\operatorname{PFS}(Y)$ & 18.4 & 6.9 & $48 \%$ \\
\hline NCT02899195 & $\begin{array}{l}\text { PrECOG } \\
\text { PrE0505 }\end{array}$ & $\begin{array}{l}\text { Cis- or Carbo-platin }+ \\
\text { Pemetrxed + Durvalumab }\end{array}$ & ॥ & $\begin{array}{l}\text { Single arm; chemotherapy + durvalumab } \\
\text { followed by durvalumab maintenance }\end{array}$ & 55 & $\operatorname{OS}(Y)$ & 21.1 & $\begin{array}{l}\text { Analysis } \\
\text { ongoing }\end{array}$ & $\begin{array}{l}\text { Analysis } \\
\text { ongoing }\end{array}$ \\
\hline NCT04334759 & DREAM3R & $\begin{array}{l}\text { Cisplatin }+ \text { Pemetrxed }+ \\
\text { Durvalumab }\end{array}$ & III & $\begin{array}{l}\text { Randomised; chemotherapy + durvalumab } \\
\text { followed by durvalumab maintenance }\end{array}$ & $\begin{array}{l}480 \\
\text { (planned) }\end{array}$ & OS & Ongoing & Ongoing & Ongoing \\
\hline NCT04153565 & $\mathrm{CCTG} / \mathrm{NCl}$ & $\begin{array}{l}\text { Cisplatin + Pemetrxed + } \\
\text { Pembrolizumab }\end{array}$ & $\| / I I$ & $\begin{array}{l}\text { Randomised } 3 \text { arm; chemotherapy }+ \\
\text { pembrolizumab vs. chemotherapy vs. } \\
\text { pembrolizumab }\end{array}$ & 520 (planned) & OS (phase III) & Ongoing & Ongoing & Ongoing \\
\hline NCT03762018 & BEAT-Meso & $\begin{array}{l}\text { Carboplatin + Pemetrexed }+ \\
\text { Bevacizumac + Atezolizumab }\end{array}$ & III & $\begin{array}{l}\text { Randomised; chemotherapy }+ \\
\text { bevacizumab vs. hemotherapy }+ \\
\text { bevacizumab }+ \text { atezolizumab }\end{array}$ & 320 (planned) & $\begin{array}{l}\text { PFS + OS (co- } \\
\text { primary) }\end{array}$ & Ongoing & Ongoing & Ongoing \\
\hline
\end{tabular}

*NCCN Guidelines. 'FDA Approved. Unless otherwise noted, chemotherapy = Cis- or Carbo- platin + Pemetrexed. MMP: Malignant pleural mesothelioma; mOS: median overall survival; PFS: progression-free survival.

Increased hemorrhage was driven predominantly by grade 1-2 epistaxis (37.4\%), aside from one grade 5 brain hemorrhage. Two toxic deaths occurred in the bevacizumab arm, compared to 0 in the chemotherapy only group, and toxicity related treatment discontinuation was significantly higher in the bevacizumab $\operatorname{arm}(24.3 \%$ vs. 6.0\%). Based on these results, bevacizumab has a category 1 designation from the NCCN in combination with cisplatin plus pemetrexed for the front line treatment of advanced or unresectable MPM, although it did not achieve any regulatory approvals ${ }^{[6,7]}$. 
More recently, encouraging data have also been reported with a VEGF monoclonal antibody plus chemotherapy combination in the second-line setting [Table 2]. The results of the phase II RAMES trial were presented at the ASCO virtual meeting in $2020^{[8]}$; patients with unresectable MPM who had progressed after standard first line chemotherapies were randomized to gemcitabine plus ramucirumab (a monoclonal antibody targeting VEGFR2) vs. gemcitabine alone. The addition of ramucirumab yielded a significant overall survival benefit, with HR of 0.70 favoring the addition of ramucirumab with a mOS of 13.8 months vs. 7.5 months with chemotherapy alone. However, patients in the RAMES trial had not received bevacizumab in the first line, and it is unclear if patients who progress on the MAPS regimen would derive similar benefit. Notably, the authors reported no increase in grade 3-4 thromboembolism or hematologic toxicity with ramucirumab $v s$. placebo; however, the authors did report an increase in grade 3-4 hypertension.

Hopes of a class effect have not borne out, as trials with VEGF TKIs have not replicated the survival benefit seen in MAPS and RAMES. In 2018, a collaborative group randomized phase II trial (SWOG S09505) examined the addition of cediranib $v s$. placebo to $\mathrm{CP}^{[9]}$. The trial met its primary endpoint of RECIST progression-free survival (PFS), with PFS increased to 7.2 months for CP plus cediranib vs. 5.6 months for $\mathrm{CP}$ plus placebo. Despite statistical significance, this PFS benefit was modest in absolute terms, and there was no corresponding difference in overall survival (10 months $v s .8 .5$ months, $P=0.44$ ). Moreover, toxicities were significantly higher in the cediranib arm including GI symptoms, hypertension, and epistaxis. Similarly, the double-blind, randomized, placebo-controlled phase III LUME-MESO trial investigated the addition of nintedanib to doublet chemotherapy. Nintedanib has been shown to target VEGF receptors, PDGF receptors $\alpha$ and $\beta$, FGF receptors 1-3, and Src and Abl kinases; it had performed well in early phase trials and the targeting of multiple related angiogenic pathways was hypothesized to increase anti-tumor efficacy. Despite encouraging data from the randomized Phase 2 portion of the trial, the Phase 3 did not meet its primary endpoint of increased PFS.

\section{INTO THE IMMUNOTHERAPY ERA}

To date, the most favorable results improving on historical chemotherapy outcomes in MPM have come from immunotherapy. The recently published Checkmate 743 trial demonstrated significant overall survival benefit with combination nivolumab/ipilimumab when compared to platinum/pemetrexed chemotherapy, leading to the first new FDA approval for the treatment of MPM in nearly 20 years ${ }^{[10,11]}$.

\section{Immunotherapy in the second-line setting}

For many years, MPM was considered to be a relatively immunologically inert tumor type; Tumor Mutational Burden, a predictor of immunotherapy outcomes in other tumor types, is generally low in $\mathrm{MPM}^{[12,13]}$.

However, the past two years have seen the maturation of practice-changing immunotherapy trials throughout multiple settings in the treatment of MPM. Early data demonstrated promise with immunotherapy for MPM, starting in the second line.

KEYNOTE-028 provided an early signal of immunotherapy activity in MPM; this study enrolled multiple disease cohorts in an open-label phase $1 \mathrm{~b}$ trial ${ }^{[14]}$. The MPM cohort enrolled 25 patients with previously treated disease and tumor PD-L1 expression $\geq 1 \%$. The safety profile was tolerable, and the authors reported a strong signal towards anti-tumor activity, with a $20 \%$ objective response rate and a further $52 \%$ of patients experiencing stable disease. Anti CTLA-4 monotherapy also exhibited strong anti-tumor activity in single arm phase II trials, with single-agent tremelimumab after progression on standard chemotherapy yielding a 
Table 2. Selected trials in the second- or third-line treatment of MPM

\begin{tabular}{|c|c|c|c|c|c|c|c|c|c|}
\hline NCT number & $\begin{array}{l}\text { Trial } \\
\text { name/Group }\end{array}$ & Drug & Phase & Design & $\begin{array}{l}\text { Number of } \\
\text { patients }\end{array}$ & $\begin{array}{l}\text { Primary endpoint } \\
(\text { Met? } \mathrm{Y} / \mathrm{N})\end{array}$ & $\begin{array}{l}\text { mOS } \\
\text { (months) }\end{array}$ & $\begin{array}{l}\text { PFS } \\
\text { (months) }\end{array}$ & $\begin{array}{l}\text { ORR } \\
\text { (RECIST) }\end{array}$ \\
\hline \multicolumn{10}{|l|}{ Chemo + VEGF } \\
\hline NCT03560973 & RAMES & Ramucirumab & ॥ & $\begin{array}{l}\text { Randomized, gemcitabine + ramucirumab vs. } \\
\text { gemcitabine + placebo }\end{array}$ & 164 & $O S(Y)$ & $\begin{array}{l}13.8 \text { vs. } 7.5 \\
(H R=0.71)\end{array}$ & 6.2 & NR \\
\hline \multicolumn{10}{|l|}{ Immunotherapy } \\
\hline NCT02054806 & KEYNOTE-028 & Pembrolizumab & $\mathrm{lb}$ & $\begin{array}{l}\text { Non-randomized, open-label, multicohort; } \\
\text { pembrolizumab }\end{array}$ & 35 & Safety $(Y)$ & 18 & 5.4 & $20 \%$ \\
\hline NCT02628067 & KEYNOTE-158* & Pembrolizumab & ॥ & $\begin{array}{l}\text { Non-randomized, open-label, multicohort; } \\
\text { pembrolizumab }\end{array}$ & 118 & ORR $(Y)$ & 10 & 2.1 & $10 \%$ \\
\hline NCT02716272 & MAPS2 ${ }^{*}$ & $\begin{array}{l}\text { Nivolumab + } \\
\text { Ipilimumab }\end{array}$ & ॥ & $\begin{array}{l}\text { Randomized, noncomparative; nivolumab vs. } \\
\text { nivolumab + ipilimumab }\end{array}$ & 125 & 12 weeks DCR (Y) & 15.9 or 11.9 & 5.6 & $29 \%$ vs. $19 \%$ \\
\hline NCT01843374 & DETERMINE & Tremelimumab & $1 \mathrm{llb}$ & Randomized; tremelimumab vs. placebo & 571 & OS $(N)$ & $\begin{array}{l}7.7 \text { vs. } 7.3 \\
(H R=0.92)\end{array}$ & 2.8 & $4.5 \%$ vs. $1.1 \%$ \\
\hline NCT02991482 & Promise-MESO & pembrolizumab & III & $\begin{array}{l}\text { Randomized; pembrolizumab vs. single agent } \\
\text { chemotherapy }\end{array}$ & 144 & $\operatorname{PFS}(\mathrm{N})$ & 10.7 vs. 12.4 & $\begin{array}{l}2.5 \text { vs. } 3.4 \\
(H R=1.06)\end{array}$ & $22 \%$ vs. $6 \%$ \\
\hline NCT03063450 & CONFIRM & $\begin{array}{l}\text { Nivolumab + } \\
\text { Ipilimumab }\end{array}$ & III & Randomized; nivolumab vs. placebo & 332 & $O S(Y)$ & $\begin{array}{l}9.2 \text { (data } \\
\text { immature) }\end{array}$ & 3 & $\begin{array}{l}\text { Analysis } \\
\text { ongoing }\end{array}$ \\
\hline
\end{tabular}

*NCCN Guidelines. MMP: Malignant pleural mesothelioma; mOS: median overall survival; PFS: progression-free survival.

$52 \%$ disease control rate, with a median duration of 10.9 months $^{[15]}$. Unfortunately, in a follow up larger, randomized phase IIb trial (DETERMINE), tremelimumab monotherapy was found to be no better than placebo. Focus then shifted to PD1/L1 inhibition and combination immunotherapy strategies ${ }^{[16]}$. A subsequent smaller non-randomized phase IIb trial using dual checkpoint blockade (tremelimumab plus durvalumab) demonstrated evidence of immune therapy efficacy, with immune-related ORR of $28 \%$ and a mOS of 16.6 month $^{[16,17]}$. Following this signal, the IFCT conducted a follow-up trial to their MAPS study, called MAPS $2^{[1]]}$. Patients progressing after first- or second-line treatment with platinum/pemetrexed based chemotherapy with or without bevacizumab were randomized to treatment with nivolumab (anti PDL-1) plus ipilimumab (anti CTLA-4) vs. nivolumab alone. The study was designed with a primary outcome of 12-week disease control, which was achieved in $40 \%$ of the ITT population treated with nivolumab independently, and in more than half (52\%) of the patients in the dual checkpoint blockade arm. Notably, an exploratory non-comparative survival analysis demonstrated a mOS of 15.9 months in the dual checkpoint blockade arm, and 11.9 months with nivolumab monotherapy, both unprecedented results in a pretreated patient population. Although objective responses by RECIST criteria were noted in $19 \%$ and $28 \%$ of patients in the nivolumab and dual checkpoint blockade arms respectively, a meaningful subgroup of patients experienced the durable progression-free disease. This appears to have driven lengthened survival, with the Kaplan-Meier curves for both PFS and OS plateauing at between 15 and 20 months with $48 \%$ of patients in the ipilimumab plus nivolumab arm still alive at data cutoff of 20.1 months. Unlike in previous trials ${ }^{[17]}$, these treatment benefits appear enhanced in patients with PD-L1 expressing tumors. In a post hoc analysis, the authors found that PD-L1 
expression in at least $1 \%$ of cells was associated with significantly higher rates of objective response to immunotherapy when assessed with both the DAKO 28-8 and SP-263 assays, and high PD-L1 expression (defined as PD-L1 $>25 \%$, measured by $28-8$ assay) was associated with an $86 \%$ disease control rate and a $71 \%$ objective response rate. Although non-comparative, this trial suggested strong rationale for immunotherapy combination over monotherapy when designing subsequent first line immunotherapy trials for MPM, based on the signal of increased efficacy in this group, and suggested that benefit was likely to be highest with PD-L1 expressing disease.

Randomized trials of PD1 inhibitors in the second-line setting have shown variable results. The phase III immunotherapy trial (PROMISE-MESO), randomized patients with relapsed or progressive unresectable MPM to receive pembrolizumab or single-agent chemotherapy (gemcitabine or vinorelbine) ${ }^{[19]}$. The study failed to demonstrate PFS or OS benefit regardless of PDL-1 status, though it did demonstrate improved response rates in the immunotherapy arm, and toxicity profile was similar. The results of the phase II KEYNOTE-158, which was reported more recently, were in line with these findings ${ }^{[20]}$. In the 118 patients with previously treated MPM who received pembrolizumab monotherapy in this trial, the authors reported a $10 \%$ ORR with mPFS of only 2.1 months, without distinction by PD-L1 expression.

On the other hand, the phase III CONFIRM trial, presented at the 2021 virtual IASLC World Conference on Lung Cancer, compared single-agent nivolumab with placebo for patients with relapsed or refractory MPM after progression with 2 or more lines of standard therapy ${ }^{[2-23]}$. The study was powered for primary endpoints of PFS and OS; although the data are still immature, the authors report that the trial has met its primary endpoints, with mOS 9.2 months with nivolumab vs. 6.6 months with placebo, (commensurate with historical survival data in untreated patients). These results appear to have been primarily driven by patients with epithelioid histology, where a hazard ratio of 0.71 favoring immunotherapy was observed. Only 13 patients enrolled on the CONFIM trial had non-epithelioid histology limiting data interpretation; however, in this cohort mOS was 5.9 months with nivolumab and 6.7 months with placebo, a difference that was not statistically significant. Notably, $96 \%$ of patients randomized received nivolumab as $\geq 3$ rd line therapy. While encouraging, the results in all second- or third-line immunotherapies may quickly become difficult to interpret, as patients receiving prior immunotherapy for MPM were excluded.

\section{First-line immunotherapy}

As alluded to above, combination nivolumab plus ipilimumab is rapidly becoming the international standard of care for eligible patients based on the results of the Checkpoint 743 phase III trial, incorporated in the NCCN guidelines with a category 1 recommendation and securing FDA approval late in $2020^{[6]}$. The trial randomized patients with relapsed or unresectable MPM without prior systemic treatment to undergo treatment with either dual checkpoint blockade (nivolumab $3 \mathrm{mg} / \mathrm{kg}$ IV every 2 weeks plus ipilimumab $1 \mathrm{mg} / \mathrm{kg}$ every 6 weeks) vs. Cisplatin or Carboplatin plus Pemetrexed at standard dosing. Bevacizumab was not included in the chemotherapy arm. The strength of the study design included its large size (> 700 patients) and the decision to power for a primary endpoint of overall survival ${ }^{[24]}$; randomization stratified for both histology (epithelioid $v s$. non-epithelioid) and gender. PDL-1 was positive (TPS $\geq 1 \%$ ) in a large proportion of patients in both arms, at $>70 \%$ of the cohort. At a prespecified interim analysis after a median follow up of 29.7 months, mOS for the immunotherapy arm was 18.1 months $v s .14 .1$ months in the chemotherapy arm, with a HR 0.74 favoring immunotherapy [Figure 1] ${ }^{[10]}$.

Unlike results in the second line CONFIRM trial ${ }^{[22]}$, substantial benefit was noted for patients with nonepithelioid tumors. Although dual checkpoint blockade outperformed chemotherapy in all randomized patients, this effect was amplified in patients with non-epithelioid tumor histology, where mOS was 18.1 


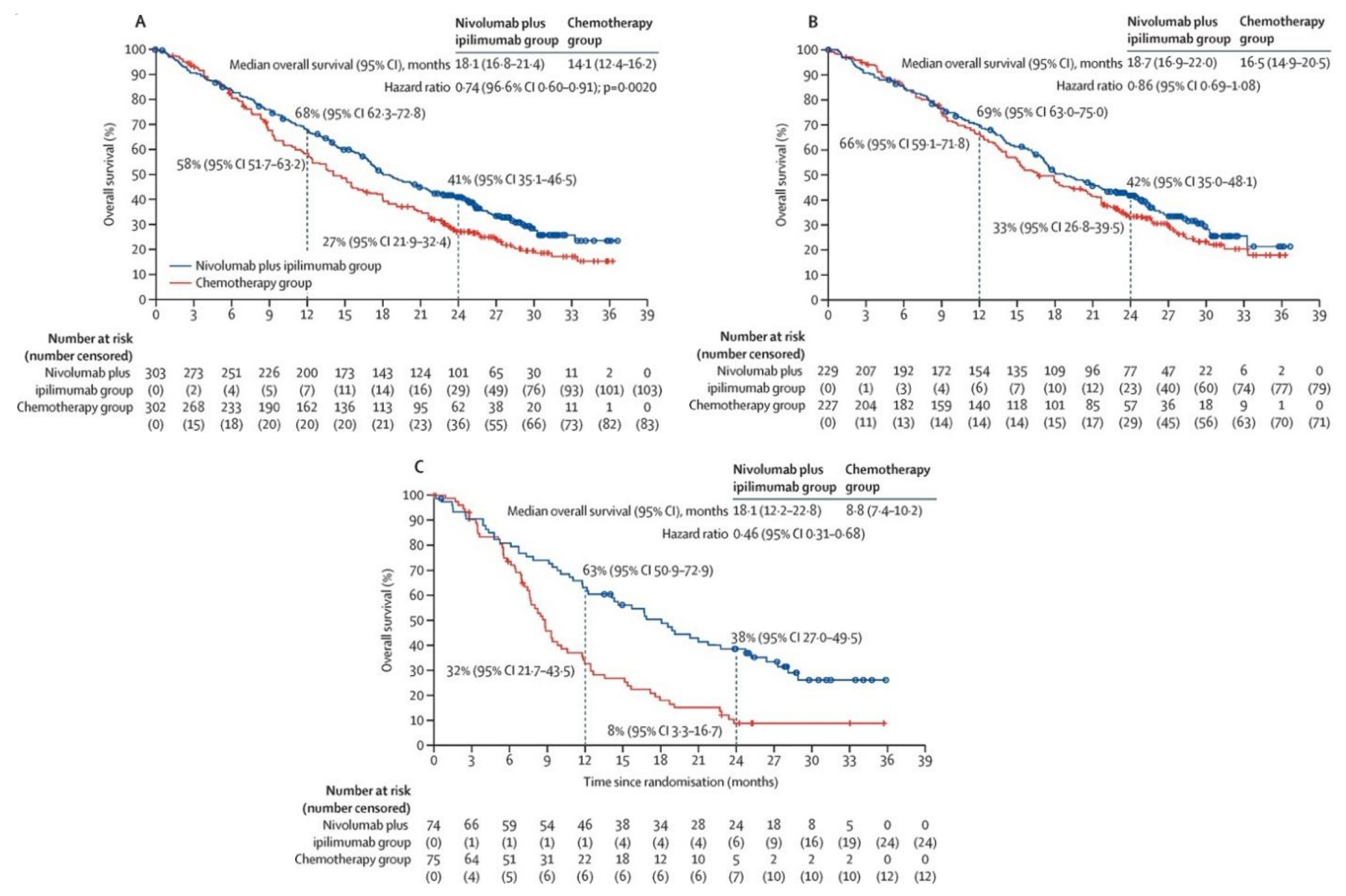

Figure 1. Results from CheckMate 743: first-line nivolumab plus ipilimumab in unresectable malignant pleural mesothelioma. Overall survival in all randomised patients (A) and in patients with epithelioid tumour histology (B) and non-epithelioid tumour histology (C). The hazard ratio in part $A$ is stratified by sex and histology. The hazard ratios in parts $B$ and $C$ are from unstratified Cox proportional hazard models. This figure is quoted with permission from Baas et al. ${ }^{[10]}$.

months vs. only 8.8 months in the chemotherapy group, and the hazard ratio for the epithelioid subgroup crossed 1 at the time of analysis. Also, of note, survival curve separation was a late effect, with survival benefit with immunotherapy only seen after 3 months of treatment. This phenomenon is likely due in large part to a number of early progressors in the immunotherapy group; progression-free survival in this cohort appears to favor the chemotherapy arm until after nearly 9 months of treatment, an important consideration for patients with rapidly progressing or highly symptomatic disease. There is also a notable flattening of the survival curves after approximately 30 months of treatment in the immunotherapy group, suggesting the potential for long-term efficacy in a subset of patients. Interpretation of outcomes by PD-L1 expression should be undertaken with caution given the small proportion of patients with low PD-L1 expression and the lack of stratification for this variable within the analysis. Overall survival outcomes were similar between the subgroups with less than $1 \%$ and with $\geq 1 \%$ PD-L1 expression.

Grade 3-4 treatment-related adverse events were comparable between the arms at $30 \%$ vs. $32 \%$ for immunotherapy and chemotherapy arms respectively. However, treatment related adverse events leading to treatment discontinuation were higher in the immunotherapy group, at $23 \% v s .16 \%$ in the chemotherapy group. The most common adverse events in the immunotherapy group were diarrhea ( $21 \%$ any grade, $3 \%$ grade 3 ), pruritis ( $16 \%$ any grade, $1 \%$ grade 3$)$, and rash ( $14 \%$ any grade, $1 \%$ grade 3 ). Treatment-related deaths were rare in both groups, with 3 treatment related deaths in the immunotherapy arm (pneumonitis, encephalitis, and heart failure) and 1 death in the chemotherapy arm caused by myelosuppression. 


\section{Chemo-immunotherapy combinations}

The success of front-line immunotherapy has raised interest in studying combination chemoimmunotherapy approaches, which hope to combine the prompt action of cytotoxic chemotherapy regimens with the durable responses of immunotherapy. This approach has been practice-changing in nonsmall cell lung cancers, where the combination of carboplatin and pemetrexed with the anti-PD-1 drug pembrolizumab has become front-line standard of care based on the results of the landmark Keynote-189 trial ${ }^{[25]}$. Early phase data in MPM are promising; The phase II US PrECOG LLC run PrE0505 study and the Australian DREAM trial each evaluated a single cohort of patients treated with combination CP chemotherapy plus the anti PDL- 1 antibody durvalumab ${ }^{[26,27]}$. PrE0505 reported a mOS of 20.4 months, with DREAM reporting a mOS of 18.4 months.

In contrast to Checkmate 743, the DREAM study enrolled primarily patients with epithelioid histology, including only 6 patients with biphasic and 1 patient with sarcomatoid histologies. In a post-hoc analysis the authors noted responses across subtypes however are careful to report that the patient with sarcomatoid disease experienced stable disease as a best radiographic response. Therefore, it is unclear based on these phase II results if the immunotherapy benefit for patients with sarcomatoid histology with ipilimumab and nivolumab will carry over to chemo-immunotherapy combinations.

Although these trials were non-comparative, the reported survival data are significantly better than what might be expected from chemotherapy alone. Moreover, the prospect of meaningful long-term benefit for significant proportion of patients is raised by these data, with nearly $40 \%$ of patients in the DREAM study alive at 2 years. These benefits appear to be agnostic of tumor PD-L1 expression. In parallel with the Checkmate 743 results, the $53 \%$ of patients with PD-L1 scores $\geq 1 \%$ demonstrated similar PFS when compared to their low PD-L1 score counterparts.

The DREAM and PrECOG LLC run PrE0505 trials had very similar designs but were performed independently in different countries. Despite this, the comparable outcomes raised hope that the findings may be further confirmed when the design is translated to a phase III comparative setting. A follow-up international phase III (DREAM3R) trial is currently recruiting, and plans to compare 4-6 cycles CP chemotherapy plus durvalumab followed by durvalumab maintenance with 4-6 cycles of CP chemotherapy followed by observation (NCT04334759). Pembrolizumab $\left(200 \mathrm{mg} / \mathrm{m}^{2} \mathrm{IV}\right.$ q3 weeks) in combination with $\mathrm{CP}$ is also being studied in an ongoing phase Ib trial (NCT04153565), and a separate phase II/III trial sponsored jointly by the Canadian Cancer Trials Group and the NCI will randomize more than 500 patients to chemotherapy, pembrolizumab monotherapy, or chemotherapy plus pembrolizumab (NCT02784171). Currently, the only ongoing trial combining immunotherapy with chemotherapy and bevacizumab is the phase III BEAT-MESO trial currently recruiting in sites across Europe which plans to complete in 2024 (NCT03762018). This trial will be powered for dual primary endpoints of progression free and overall survival and will randomize patients to receive atezolizumab $1200 \mathrm{mg}$ IV plus bevacizumab $15 \mathrm{mg} / \mathrm{kg}$ with carboplatin plus pemetrexed every 3 weeks $v s$. chemotherapy plus bevacizumab alone.

\section{CONCLUSION AND FUTURE DIRECTIONS}

The breadth and scope of clinical trials which have recently been reported and are currently ongoing for such a rare disease as MPM provide reason for optimism. The success of several large-scale well-designed trials proves that ambitious clinical trials to answer urgent questions about the treatment of MPM are not only exigent but feasible, and there is reason to believe that outcomes for patients with MPM will continue to improve rapidly over the next decade. OS remains the gold standard for establishing benefit, as ORR and PFS are difficult to measure in MPM and may not correlate as evidenced from phase I-II studies that have 


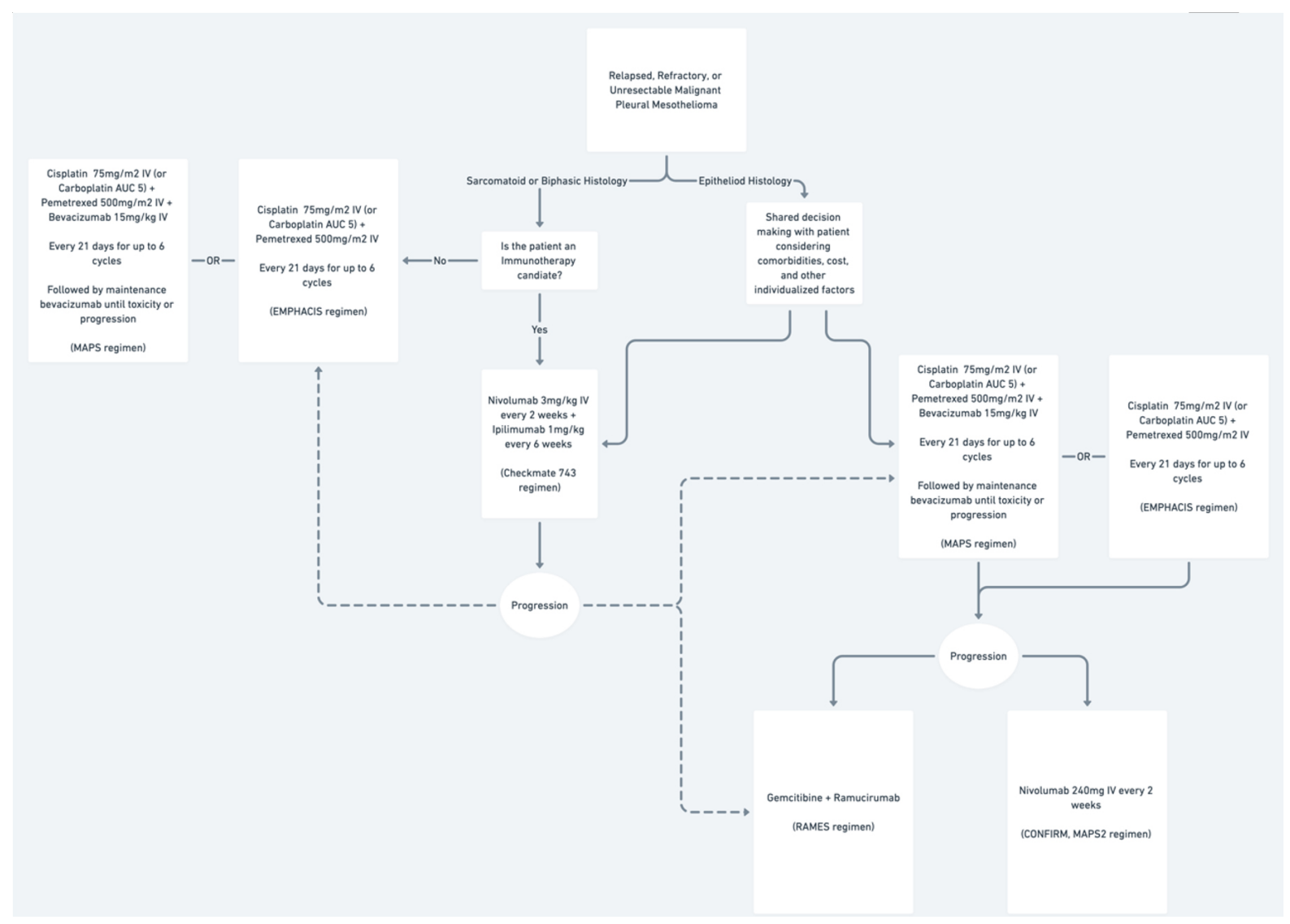

Figure 2. A proposed framework for the evidence-based treatment of unresectable malignant pleural mesothelioma.

not been borne out in the phase III setting.

Options for the treatment of unresectable MPM in both the front-line and subsequent-line settings remain limited. However, the additions of robust data in immunotherapy and supplemental anti-VEGF treatments to traditional chemotherapy have unquestionably changed the standard of care. Clinicians are now able to choose between standard chemotherapy, chemotherapy plus bevacizumab, or nivolumab plus ipilimumab as first line treatments for unresectable malignant pleural mesothelioma [Figure 2].

Future trials will build on immunotherapy backbones. Despite representing a significant advance, the objective response rate of $40 \%$ with dual checkpoint blockade seen in Checkmate 743 leaves the majority of patients without response to treatment ${ }^{[10]}$. Efforts to increase efficacy by adding additional agents are highly anticipated. The phase II data for chemo-immunotherapy combinations has been exceptional, and is already infiltrating clinical practice in selected circumstances, although notably phase II data have often not translated to positive phase III trials in MPM, as seen with Nintedanib and other anti-VEGF agents ${ }^{[28]}$. As immunotherapy solidifies its place within front-line treatment, second-line trials examining the response rates to chemotherapy with or without bevacizumab after front line ipilimumab plus nivolumab will be needed to determine optimal sequencing of treatments, and combinations with other novel agents will hopefully further improve outcomes. 
Although outside of the scope of this review, other novel approaches under investigation seek to capitalize on increasing knowledge of MPM biology and its immune microenvironment. Anti-tumor vaccines are in development and are under early phase investigation in combination with checkpoint inhibition (NCT04040231). CAR-T therapies targeting the cell surface antigen mesothelin also represent an exciting area of innovation, and may be used to potentiate anti-tumor effect of immune checkpoint inhibitors in future studies (NCT02414269, NCT03126630).

There is also a large unmet need to define optimal treatment in patients with poor performance status (ECOG 2) as these patients have been universally excluded from the trials which have to date lead to FDA approvals. Finally, there also remains a significant unmet need to define biomarkers that can reliably identify patients who can benefit disproportionately from immunotherapy or other targeted therapies.

\section{DECLARATIONS}

\section{Authors' contributions}

Made substantial contributions to conception and writing of the article: Fitzgerald BG, Krug LM

\section{Availability of data and materials}

Not applicable.

\section{Financial support and sponsorship}

None.

\section{Conflicts of interest}

Lee M. Krug: AstraZeneca employee and stock holder.

\section{Ethical approval and consent to participate}

Not applicable.

\section{Consent for publication}

Not applicable.

\section{Copyright}

(c) The Author(s) 2021.

\section{REFERENCES}

1. Vogelzang NJ, Rusthoven JJ, Symanowski J, et al. Phase III study of pemetrexed in combination with cisplatin versus cisplatin alone in patients with malignant pleural mesothelioma. J Clin Oncol 2003;21:2636-44. DOI PubMed

2. Ohta Y, Shridhar V, Bright RK, et al. VEGF and VEGF type C play an important role in angiogenesis and lymphangiogenesis in human malignant mesothelioma tumours. Br J Cancer 1999;81:54-61. DOI PubMed PMC

3. Strizzi L, Catalano A, Vianale G, et al. Vascular endothelial growth factor is an autocrine growth factor in human malignant mesothelioma. J Pathol 2001;193:468-75. DOI PubMed

4. Zalcman G, Margery J, Scherpereel A, et al. IFCT-GFPC-0701 MAPS trial, a multicenter randomized phase II/III trial of pemetrexedcisplatin with or without bevacizumab in patients with malignant pleural mesothelioma. JCO 2010;28:7020. DOI PubMed

5. Kindler HL, Karrison TG, Gandara DR, et al. Multicenter, double-blind, placebo-controlled, randomized phase II trial of gemcitabine/cisplatin plus bevacizumab or placebo in patients with malignant mesothelioma. J Clin Oncol 2012;30:2509-15. DOI PubMed PMC

6. National Comprehensive Cancer Network. Malignant Pleural Mesothelioma (Version 2.2021). Available from: https://www.nccn.org/professionals/physician_gls/pdf/mpm.pdf. [Last accessed on 4 Aug 2021].

7. Genentech Halts Avastin Approval Filing for Mesothelioma. Available from: https:/www.curemeso.org/2017/06/28/genentech-haltsavastin-filing-mesothelioma/. [Last accessed on 4 Aug 2021].

8. Pagano M, Ceresoli GL, Zucali PA, et al. Randomized phase II study on gemcitabine with or without ramucirumab as second-line treatment for advanced malignant pleural mesothelioma (MPM): Results of Italian Rames Study. J Clin Oncol 2020;38:9004. DOI

9. Tsao AS, Miao J, Wistuba II, et al. SWOG S0905: A randomized phase II study of cediranib versus placebo in combination with 
cisplatin and pemetrexed in chemonaive patients with malignant pleural mesothelioma. J Clin Oncol 2018;36:8514. DOI

10. Baas P, Scherpereel A, Nowak AK, et al. First-line nivolumab plus ipilimumab in unresectable malignant pleural mesothelioma (CheckMate 743): a multicentre, randomised, open-label, phase 3 trial. Lancet 2021;397:375-86. DOI PubMed

11. FDA Approves Drug Combination for Treating Mesothelioma. Available from: https://www.fda.gov/news-events/pressannouncements/fda-approves-drug-combination-treating-mesothelioma. [Last accessed on 4 Aug 2021].

12. Samstein RM, Lee CH, Shoushtari AN, et al. Tumor mutational load predicts survival after immunotherapy across multiple cancer types. Nat Genet 2019;51:202-6. DOI PubMed PMC

13. Markowitz P, Patel M, Groisberg R, et al. Genomic characterization of malignant pleural mesothelioma and associated clinical outcomes. Cancer Treat Res Commun 2020;25:100232. DOI PubMed

14. Alley EW, Lopez J, Santoro A, et al. Clinical safety and activity of pembrolizumab in patients with malignant pleural mesothelioma (KEYNOTE-028): preliminary results from a non-randomised, open-label, phase 1b trial. Lancet Oncol 2017;18:623-30. DOI PubMed

15. Calabrò L, Morra A, Fonsatti E, et al. Efficacy and safety of an intensified schedule of tremelimumab for chemotherapy-resistant malignant mesothelioma: an open-label, single-arm, phase 2 study. Lancet Respir Med 2015;3:301-9. DOI PubMed

16. Maio M, Scherpereel A, Calabrò L, et al. Tremelimumab as second-line or third-line treatment in relapsed malignant mesothelioma (DETERMINE): a multicentre, international, randomised, double-blind, placebo-controlled phase 2b trial. Lancet Oncol 2017;18:1261-73. DOI PubMed

17. Calabrò L, Morra A, Giannarelli D, et al. Tremelimumab combined with durvalumab in patients with mesothelioma (NIBIT-MESO-1): an open-label, non-randomised, phase 2 study. Lancet Respir Med 2018;6:451-60. DOI PubMed

18. Scherpereel A, Mazieres J, Greillier L, et al. Nivolumab or nivolumab plus ipilimumab in patients with relapsed malignant pleural mesothelioma (IFCT-1501 MAPS2): a multicentre, open-label, randomised, non-comparative, phase 2 trial. Lancet Oncol 2019;20:239-53. DOI PubMed

19. Popat S, Curioni-Fontecedro A, Dafni U, et al. A multicentre randomised phase III trial comparing pembrolizumab versus single-agent chemotherapy for advanced pre-treated malignant pleural mesothelioma: the European Thoracic Oncology Platform (ETOP 9-15) PROMISE-meso trial. Ann Oncol 2020;31:1734-45. DOI PubMed

20. Yap TA, Nakagawa K, Fujimoto N, et al. Efficacy and safety of pembrolizumab in patients with advanced mesothelioma in the openlabel, single-arm, phase 2 KEYNOTE-158 study. Lancet Respir Med 2021;9:613-21. DOI PubMed

21. Fennell DA, Kirkpatrick E, Cozens K, et al. CONFIRM: a double-blind, placebo-controlled phase III clinical trial investigating the effect of nivolumab in patients with relapsed mesothelioma: study protocol for a randomised controlled trial. Trials 2018;19:233. DOI PubMed PMC

22. Fennell D, Ottensmeier C, Califano R, et al. Nivolumab versus placebo in relapsed malignant mesothelioma: preliminary results from the CONFIRM phase 3 trial. 2020 World Conference on Lung Cancer Singapore 2021. DOI

23. Fennell D, Ottensmeier C, Califano R, et al. PS01.11 nivolumab versus placebo in relapsed malignant mesothelioma: the CONFIRM phase 3 trial. J Thorac Oncol 2021;16:S62. DOI

24. Zalcman G, Peters S, Mansfield AS, et al. Checkmate 743: A phase 3, randomized, open-label trial of nivolumab (nivo) plus ipilimumab (ipi) vs pemetrexed plus cisplatin or carboplatin as first-line therapy in unresectable pleural mesothelioma. J Clin Oncol 2017;35:TPS8581. DOI

25. Gandhi L, Rodríguez-Abreu D, Gadgeel S, et al; KEYNOTE-189 Investigators. Pembrolizumab plus chemotherapy in metastatic nonsmall-cell lung cancer. N Engl J Med 2018;378:2078-92. DOI PubMed

26. Forde, P. M., et al. PrE0505: Phase II multicenter study of anti-PD-L1, durvalumab, in combination with cisplatin and pemetrexed for the first-line treatment of unresectable malignant pleural mesothelioma (MPM)-A PrECOG LLC study. J Clin Oncol 2020;38:9003. DOI

27. Nowak AK, Lesterhuis WJ, Kok P, et al. Durvalumab with first-line chemotherapy in previously untreated malignant pleural mesothelioma (DREAM): a multicentre, single-arm, phase 2 trial with a safety run-in. Lancet Oncol 2020;21:1213-23. DOI PubMed

28. Scagliotti GV, Gaafar R, Nowak AK, et al. Nintedanib in combination with pemetrexed and cisplatin for chemotherapy-naive patients with advanced malignant pleural mesothelioma (LUME-Meso): a double-blind, randomised, placebo-controlled phase 3 trial. Lancet Respir Med 2019;7:569-80. DOI PubMed 